\title{
A Tablet PC based System for Ubiquitous Patient Monitoring and Smart Alert Generation in an Intensive Care Unit
}

\author{
Joms Antony \\ Department of Information Technology \\ Amal Jyothi College of Engineering \\ Kottayam-686518, India
}

\author{
Anju K.S \\ Department of Computer Science \& Engineering \\ Amal Jyothi College of Engineering \\ Kottayam-686518, India
}

\author{
Anumol Mathew \\ Department of Computer Science \& Engineering \\ Amal Jyothi College of Engineering \\ Kottayam-686518, India
}

\begin{abstract}
Providing critical care to patients admitted to an ICU is a very important aspect in healthcare. This requires mechanisms for ubiquitous monitoring and smart alert generation systems. The conventional systems in many hospitals are not able to cope up with this requirement. So that medication and other required actions will be getting delayed making life difficult for a patient. These issues can be addressed by implementing a Tablet PC based system focussed on ICU based work flows. Designing and developing of Apps can provide better solutions for clinical data entry, patient monitoring and meaningful alert generation. The clinical data entry can be made easier with the help of a Tablet PC which offers a number of possibilities for organised data entry. Once the data becomes available; forms, charts, graphs etc can be generated easily without much manual intervention. Interfacing bed side monitors to the central server can provide automated and ubiquitous solutions for monitoring and analysis of patient data. Another important aspect is the way in which alert generation is made. In existing systems alerts are generated based on hard limits set by the clinical staff. There is a need to develop a more comprehensive system that holistically looks at all available information about the situation before generating an alert. Towards this, it should continuously monitor all vital signals available and evaluate the cross functional effects between them before arriving at the seriousness of the conditions leading to more meaningful alerts.
\end{abstract}

\section{Keywords:}

Critical Care, Device Interfacing, Smart Alerts, Decision Support, Android Apps, Hospital Information Management Systems (HIMS).

\section{INTRODUCTION}

Most of the conventional Hospital Information Management Systems (HIMS) make use of stationary Desktop PCs for work flow automation. This has lot of limitations with regard to the clinical data entry, patient monitoring and generation of Electronic Medical Records. The use of Desktop PCs results in lot of difficulties which possibly can be avoided if Tablet PCs are deployed. These Tablet PCs are centrally connected to a server in a Wi-Fi enabled hospital space. This system offers a set of advantages for the clinical staff, since they can move around and perform various tasks and at the same time use the device at their point of service. It will reduce the effort involved in clinical data entry, thereby allowing them to provide better patient care. The system will automatically provide more accuracy, swiftness and flexibility to their work flow.

The clinical staff is involved in various activities like; monitoring and recording of patients vital signs, generating charts and progress notes, issuing lab request and retrieving the results etc. All these can be performed easily by making use of independent applications. The authors are in the process of developing separate apps for the activities mentioned above. These applications are being developed using the android platform which is the de-facto standard for mobile application development. Android provides a single application model that enables the deployment across a wide range of devicesfrom phones to tablets and beyond. Android also gives tools for creating apps that look great and take advantage of the hardware capabilities available on each device.

One of the most important activities in a critical care unit is the monitoring of vital signs (ECG, Blood Pressure, Dissolved Oxygen Content and Respiration Rate) and other parameters of the patient. In the existing scenario these parameters are recorded manually and are re-entered into HIMS. This kind of recording system causes a lot of overheads. The clinical staff will have to run around patient beds to collect these data and has to make a paper based manual entry which is replicated and fed to the conventional HIMS.

In this system most of the patient monitoring devices [1] is connected to a central server which can automatically record the various patient parameters and store it in a database. Now this data becomes centrally available and can be accessed by an authorised Wi-Fi connected Tablet PC inside the hospital space. Since the system implemented as a client-server model, the services of the system is also open to internet connected devices. Here GPRS can be the mode of communication and smart phones can act as remote clients.

The team is developing an alert generation system based on continuous monitoring of the parameters captured by the Starplus 


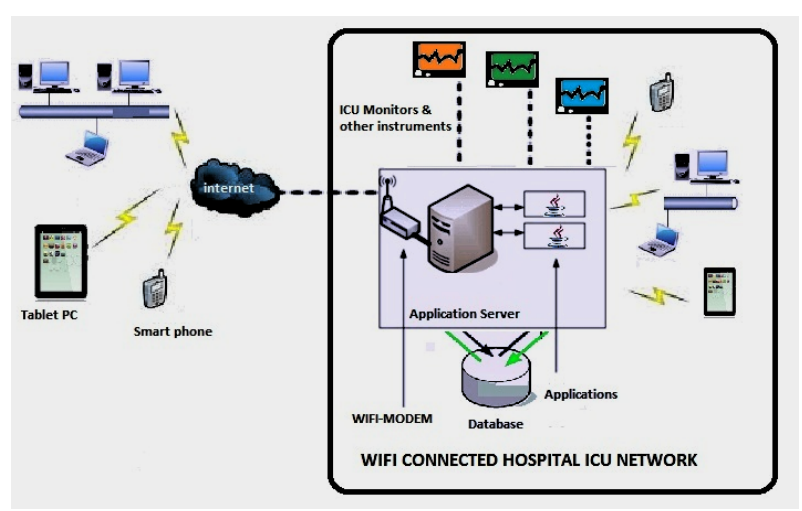

Fig. 1. ICU Automation System Architecture.

300 bed-side monitoring system in the Medical Neuro-ICU of Jubilee Mission Medical College in Trichur. In this project the issue of alert generation in a critical care unit is also addressed. In the existing system the alert generation happens based on predefined hard limits (upper/lower) set in the monitoring devices. Here the alert generation is static and is not meaningful. The team is working towards a dynamic alert generation which is different from the conventional static alert generation. The system consists of a decision support module which takes into account the different patient parameters on a real-time basis, analyse their trends and can generate alerts based on a context aware rule set.

This paper presents the salient features of the system developed. It is organized as follows: Section II describes the overall scheme of the ICU automation system with special emphasis to the views available to the clinicians. Section 3 describes a nutshell view of the different apps being developed to support clinical data entry, automatic patient monitoring, generation of electronic health records etc. This section also describes about the application for making a lab request and retrieving lab investigation reports which was found to be very useful. Section 4 describe the features of multipara patient monitoring systems, particularly about Starplus 300 monitoring station, the parameters monitored, the interfacing to the ICU server and the method of signal collection. Section 5 describes about the dynamic smart alert generation making of context aware rule set. Section 6 provides an impact analysis of this system from the perspective of the nursing staff and the clinicians

\section{ICU AUTOMATION SYSTEM}

The architecture of the ICU Automation system is as depicted in figure 1. The ICU Automation system is designed in such a way that there is a central server where the database and applications are deployed. A client -server architecture allows a seamless flow of information to everyone in the clinical team. Thereby supporting efficient work flow and immediate access to reliable patient information where it is needed most. This kind of automation organizes high volume device data, lab results, calculations, orders, medications and clinical documentation for physicians, nurses and other members of the hospital team. Thus the work flow can be made easier and informative. The ICU Server is also connected to the main server via the hospital network as shown in figure 2 .

A web enabled application allows access to the system from a remote location. This will help a physician to be aware of the condition of the patient well in advance. One of the greatest impacts of this system is that all the clinical information is stored in the server and is available to the different members of the clinical team based on their hierarchies. There is a vast amount of
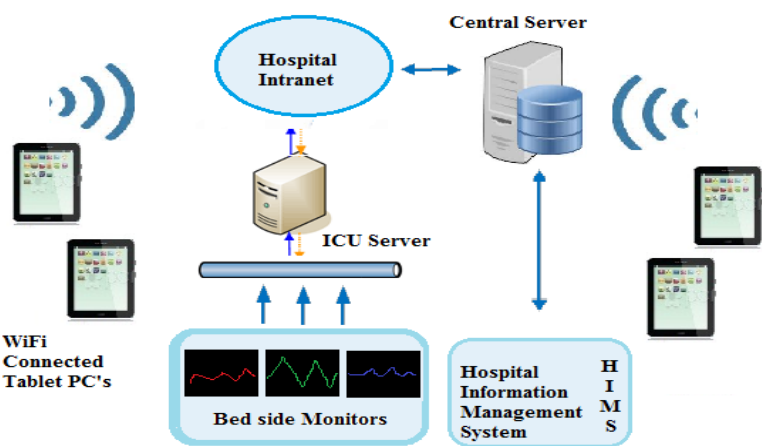

Fig. 2. Network Architecture.

data entry associated with different clinical staffs in the form of charts and records like

\section{- History and physical record \\ -Monitoring of vital signs \\ -Gaphical charts \\ -Medication record \\ -Patient progress notes \\ -Prescription orders \\ —Nurses record \\ —Laboratory request/reports}

The paper based clinical data entry is very hectic and can reduce the amount of care provided to the patients. On careful examination it can be identified that most of the data entry is redundant. The entry of clinical data into the system can be done in various ways depending on the way in which the information is collected. Interactive forms, dropdown lists, templates etc can be used for making data entry easier. The use of tablet PCS also allows to record voice, take photographs, upload files and images which can be extremely useful.

Another advantage of the system is that it can directly read from equipments connected to the patient like multi-para monitors, ventilators etc. This can be made possible by establishing a proper communication interface between equipments and the server system by using communication ports. Some of the equipment manufacturers also provide a central monitoring station using which the patient at each ICU bed can be monitored. Eg: starplus multi-para monitor which is described in the next section.

In the proposed architecture, there is a WIFI connected ICU space [2] where the physicians and nurses will be provided with a tablet PC which can be used for data entry into the system. This allows faster, accurate and easier entry of data [3] [4] into the system. Once the data is entered properly the different faceted views can be provided to the end users (doctors, clinical staffs and others). Thus the end users can analyze the patient within few clicks by reviewing his EHR(Electronic Health Record) information.

In the system Implementation open source software and open source hardware utilities are used. The ICU server has its own database and application servers deployed in it. PostGreSQL serves as the DBMS engine and JBOSS provides the application server functionality. Hybernate provides the DBMS connectivity and Spring framework is used for application development at server side. The Server OS used is Ubuntu Server Edition 
Table 1. Details of data collection in an ICU

\begin{tabular}{|c|l|l|}
\hline Activity & Equipments used & Method of data Entry \\
\hline Monitoring Vital signs & $\begin{array}{l}\text { multipara patient moni- } \\
\text { tor,pulse oximeter mon- } \\
\text { itor }\end{array}$ & $\begin{array}{l}\text { Through machine inter- } \\
\text { face }\end{array}$ \\
\cline { 2 - 3 } & $\begin{array}{l}\text { Thermometer, blood } \\
\text { pressure cuff and } \\
\text { stethoscope }\end{array}$ & $\begin{array}{l}\text { Manually entered us- } \\
\text { ing wif connected } \\
\text { tabletPC/Smartphone }\end{array}$ \\
\hline Fluids(in/out) & Measuring instruments & $\begin{array}{l}\text { Manually entered us- } \\
\text { ing wifi connected } \\
\text { tabletPC/Smartphone }\end{array}$ \\
\hline Laboratory data & Medical lab equipments & $\begin{array}{l}\text { Automatic or manual } \\
\text { based on the equip- } \\
\text { ments }\end{array}$ \\
\hline
\end{tabular}

\section{APPS FOR WORK FLOW AUTOMATION}

In an ICU various systems are used for collecting different data [6]. These systems include medical devices, laboratory systems, patients demographics, EMR (Electronic Medical Record), pharmacy system and end users. There exist different clinical data which are real time variables. These variables are entered automatically or manually to the system. The real time data include ventilator and monitor data, other device data etc can be fed to the system automatically where as static clinical data have to be entered manually. The details of data collection in an ICU is shown in Table. 3. On careful analysis it can be found that there is scope for automating these activities which in turn can generate the views of each patient in systematic manner.

Individual Apps running on a tablet PC can bring in freedom in time, space and convenience. They offer lot of inbuilt features like image capture, voice recording, file uploading, video streaming etc which will be very useful and handy for the clinical staff. This section discusses some of the Apps which are being designed and developed to run on a tablet PC. Here some Apps which will be very useful in patient vital signs monitoring, generation of progress charts and EMR, Lab Request and Investigation Reports etc will be discussed. These Apps can be individually invoked by clicking on relevant icon. These icons will be designed by using images and appropriate names which will clearly reveal their purpose. Each of these icons will trigger a simple activity which can be easily understood and executed by an end user. These simple activities will update the central database whenever necessary and will be able to generate views and reports whenever necessary.

These activities and their work flow using some screen shots. Some of the Apps are listed below and are discussed in detail.

(1) Patient Monitor

(2) ICU Direct Monitor

(3) Vitals Chart

(4) Lab Wizard

\subsection{Patient Monitor}

This App is used for the overall monitoring of an ICU patient. The doctors or the clinical staff can easily review a patient selected by his bed number. The different review facets include charts, documents, images, lab results etc. The different iconic interfaces are carefully designed which will allow an end user to collect the required information within a few taps. A sample screenshot is shown in figure 3 .

\subsection{ICU Direct Monitor}

This App is used particularly for live monitoring of vital signs without coming to the bedside. This is made possible by interfac-

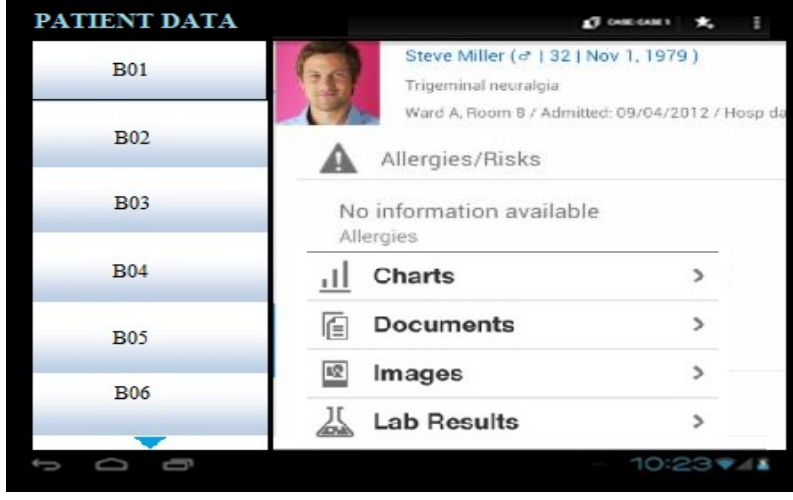

Fig. 3. A View of Patient Data Application

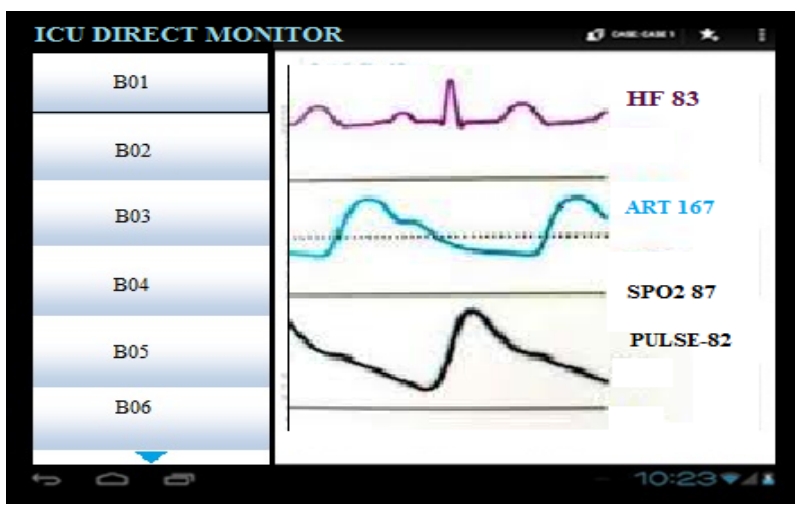

Fig. 4. ICU Direct Monitor Application.

ing the patient monitoring devices like multipara-monitors and other instruments to the central ICU server. Interfacing some of these monitoring devices are possible but not all. The issue here is that there are different makes and models of devices available in in a single ICU each of which has got its own protocols for data communication. Most of the modern devices have common protocols and TCP/IP interfaces and thus can be configured without much difficulty. Once the interfacing is done plotting real time graphs corresponding to patient vital signs can be done using the ICU Direct Monitor App as shown in figure 4.

\subsection{Vitals Chart}

This App is used for hourly recording of vital signs and for providing a chart view to the consulting clinicians. This App had a major impact by making life easier for ICU nursing staff. In the conventional system the nursing staff will have to run around different beds to collect the reading and again re-enter these to the system/forms.

Once this App was in place this became very easier for them since they can move around and make the entries which will be directly captured to the system. Now Consulting Doctors can view this chart by selecting the corresponding bed number as shown in figure 5 .

\subsection{Lab Wizard}

This App is used to issue lab test request and retrieve the details of investigation results. This App took away a lot of overhead involved when it was done manually. The procedure for requesting for a lab test and retrieving results is as shown in figure 6 . The work flow is clearly mentioned in the figure. The following steps are involved in this procedure. 


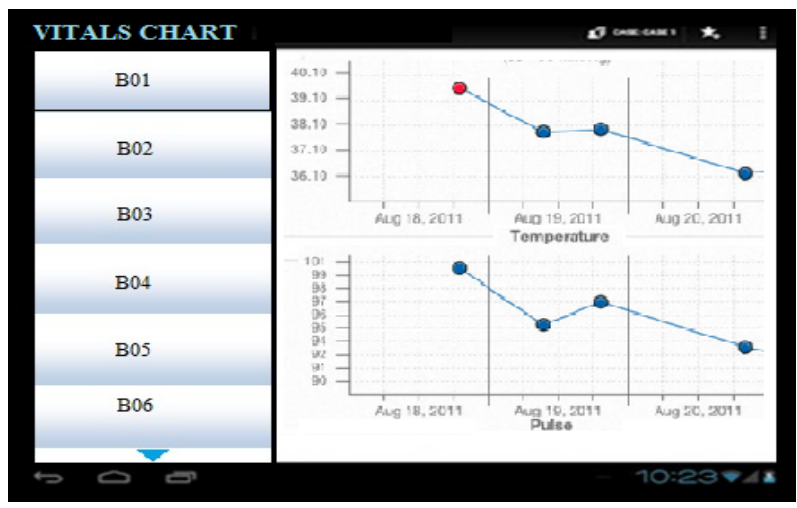

Fig. 5. A View of Vitals Chart.

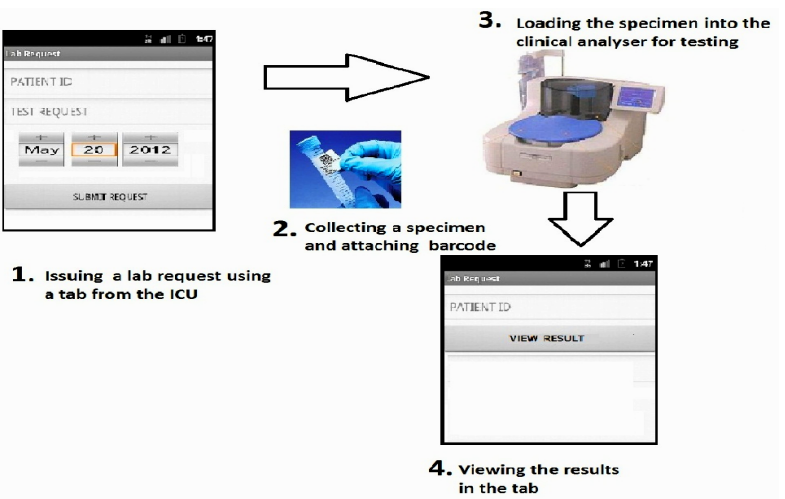

Fig. 6. Requesting for a lab test and retrieving results.

Issuing a lab request: This is done by the duty nurses using a Tablet PC by invoking the Lab request activity designed in the application

Collecting the Specimen: The duty nurse collects the specimen as per the request and attaches a barcode for identification purpose.

Loading the specimen: The lab assistant loads the specimen into the clinical analyser for testing which will dynamically generate the results and feeds it into the system. The system can identify the patient-specimen association from the barcode.

Viewing the results: The doctors, nurses and other clinical staff can view the result as and when it is available using the View result activity designed in this application.

\section{MULTI-PARAMETER PATIENT MONITORING SYSTEMS}

Multi-parameter patient monitor is used in hospital ICUs for monitoring the vital signs of patients such as ECG, Temperature(TEMP), Non-Invasive Blood Pressure(NIBP), Heart rate, Pulse Oxygen Saturation $\left(\mathrm{SPO}_{2}\right)$, Respiration rate(RESP) etc.

\subsection{Starplus Multipara Monitor and its Features}

The Starplus multi-para monitor is one such electronic equipment used to monitor the above mentioned parameters. It can offer high resolution graphical/tabular outputs which are suitable for accurate monitoring as shown in figure 7 . These multipara monitors are easily upgradable and are available in different

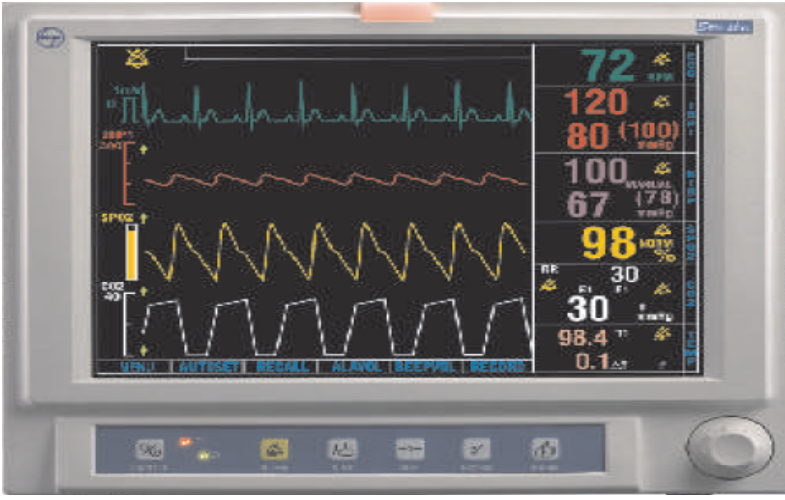

Fig. 7. Starplus Multi-para Monitor indicating ECG, SPO2, NIBP, RESP, TEMP.

screen sizes. They also have provisions for user-friendly features like audio and visual alarms.

The salient features of this device are as follows

-Upgradable 4 channel monitor with in-built 10.4"TFT bigscreen, real-color, wide-visual-angle and high brightness display

-Able to measure in-phase 3-7-channel ECG, Temperature, Non-invasive Blood Pressure, Heart Rate, Respiration and Pulse Conduction time.

-Optional dual channel thermal array recorder

-24 hrs. graphical and tabular trends with zoom facility(non volatile)

- Graded and colour coded alarms with auto-limit settings(2 stages)

- Critical alarm review page

-In built with chargeable, maintenance-free and high-capacity batteries, thus able to work more than 2 hours without additional power supply.

-Wired and Wireless Central Nurses' Station software can be used for

-Multiparameter mode for detailed view of any bed

-Multi-bed mode for simultaneous view

-Upto $72 \mathrm{hrs}$ of full waveform disclosure

-Manual printing and alarm trigger printing

-Manual printing and alarm trigger printing

The various patient parameter signals can be accessed from these monitoring devices through communication ports like RS422, RS232 and can be stored in the server database in the required format. In some cases there are ports which will output analogue values(Micro//Milli volts) corresponding to parameters like ECG, temperature which can be decoded and can be collected via serial port and can be logged into the database or a text file.The open hardware platforms [13] were used for this purpose. The arduino uno board was used for this purpose. There was an SD card shield mounted on this board to transfer these values into a text file created and saved in the SD card, subsequently the values were fed to the database. This process of data logging using the arduino board is depicted in the figure 8

Once these values are stored in a server database, it will be possible to analyse the trends which in turn can be used for predictions and classifications like outset of stroke, fever etc.

Most of the existing vital-signs monitoring systems have alarm generation provisions, but these are based on predefined thresholds (upper limit/lower limit) [10 set by the technician as per doctors advice. In this scenario the clinical team is alerted only 


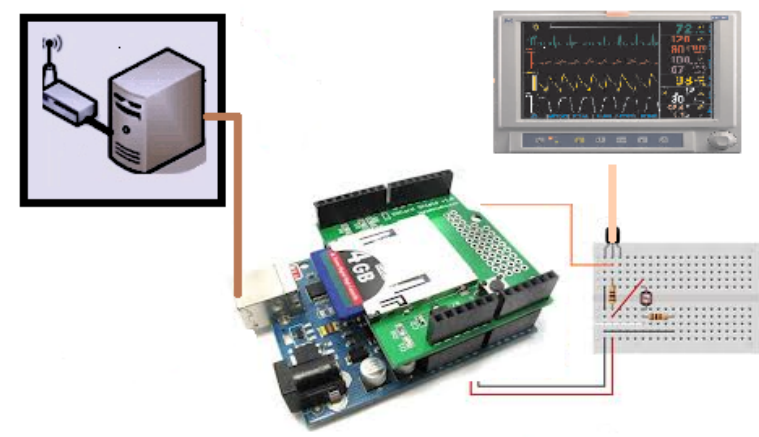

Fig. 8. Parameter data logging using the arduino board.

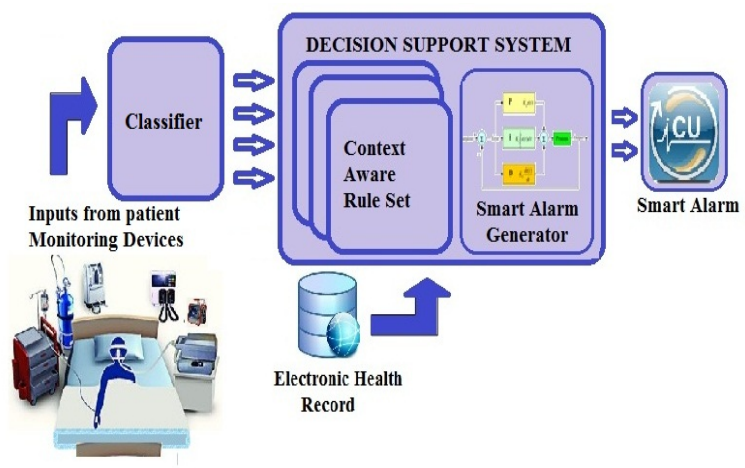

Fig. 9. Dynamic Alert level Determination-System Architecture.

when these hard limits are crossed. Here there is no scope for trends and combinatory parameter analysis [5]. This issue is discussed in the next section.

\section{DYNAMIC ALERT GENERATION}

As discussed in section 3 in conventional monitoring systems there is no scope for trends and combinatory parameter analysis. Thus the alert generation in existing systems is done in a static manner. In the proposed system provisions for dynamic alert determination which will generate alarms more meaningfully will be incorporated. The architecture of the system is as shown in figure 9. Here the vital signs (heart rate, blood pressure, blood oxygen saturation, and respiratory rate )from monitoring devices are fed to a classifier [7] module which can classify these signals into different labels like (Low, Normal ,High ,Very High etc.). The classifier output is fed to a decision support system [8] [11] which consists of a smart alarm generator [9] and a context aware rule set. The decision support system also collects inputs from electronic health records which can provide useful information regarding potential drug interactions and specific patient physiological parameters.

The smart alarm generator is basically a controller module that will analyze the parameter signals and identify trends like decreasing, increasing, shooting-up, dropping etc. The smart alarm controller makes use of a rule set [12] which is a collection of rules defined by experts in medical domain. These rules can also be customized by a doctor based on a particular patient scenario. An example for such a rule is as shown below.

IF $<$ Pulse rate increasing $>$ AND

$<$ Systolic blood pressure is decreasing >AND

<Serum potassium level is increasing>
Table 2. Hourly Patient Monitoring Data

\begin{tabular}{|c|c|c|c|c|}
\hline $\begin{array}{l}\text { Timeline } \\
\text { hour }\end{array}$ & $\begin{array}{l}\text { Systolic } \\
\text { blood } \\
\text { Pressure }\end{array}$ & $\begin{array}{l}\text { Diastolic } \\
\text { blood } \\
\text { Pressure }\end{array}$ & Pulse rate & $\begin{array}{l}\text { Serum } \\
\text { pottasium }\end{array}$ \\
\hline $\mathbf{4}$ & 130 & 90 & 80 & 4.5 \\
\hline $\mathbf{5}$ & 135 & 85 & 85 & 4.5 \\
\hline $\mathbf{6}$ & 110 & 80 & 90 & 4.5 \\
\hline $\mathbf{7}$ & 100 & 75 & 110 & 5.0 \\
\hline $\mathbf{8}$ & 90 & 70 & 120 & 5.5 \\
\hline $\mathbf{9}$ & 90 & 60 & 120 & 5.5 \\
\hline $\mathbf{1 0}$ & 90 & 60 & 130 & 5.5 \\
\hline $\mathbf{1 1}$ & $\mathbf{8 5}$ & $\mathbf{6 0}$ & $\mathbf{1 3 0}$ & 5.8 \\
\hline $\mathbf{1 2}$ & 85 & 50 & 130 & 5.9 \\
\hline $\mathbf{1 3}$ & 85 & 50 & 130 & 6.5 \\
\hline $\mathbf{1 4}$ & 80 & 50 & 130 & 6.5 \\
\hline $\mathbf{1 5}$ & 80 & 50 & 130 & 6.6 \\
\hline $\mathbf{1 6}$ & 70 & 50 & 130 & 6.8 \\
\hline $\mathbf{1 7}$ & $\mathbf{7 0}$ & $\mathbf{5 0}$ & $\mathbf{1 3 0}$ & 6.8 \\
\hline $\mathbf{1 8}$ & 100 & 50 & 130 & 6.2 \\
\hline
\end{tabular}

THEN

<Alert: Patient blood pressure is dropping>

<Treatment: Increase fluid amount>

The table 5 shown depicts the variations in vital signs of a patient on an hourly basis. Here a conventional monitoring system generates an alarm for systolic blood pressure drop only at the 17th hour. The dynamic alert generation system monitors the trends of others parameters like diastolic blood pressure, pulse rate and serum potassium. Now based on the rule shown above, the system will be able to identify that systolic blood pressure is going to drop at 11th hour itself. So an alert can be generated at 11th hour to the care taker so that she can administer the treatment as per specifications.

The alerts generated can be classified into different categories based on its criticality. The delivery of these alerts to the different hierarchies of clinical staff is taken care by the smart alarm generation system. The smart alarm generator can analyse the seriousness of the alert and can deliver it to the appropriate hierarchies depending upon their context.

\section{IMPACT ANALYSIS}

The system once developed completely, will considerably improve the effectiveness of the current workflow. It will provide

—Easier and Improved analysis of patient history

-Improved patient care

-Efficient and flexible work flow

-Easy Documentation and Record Keeping

-Real-time monitoring with time stamping

-Enables research and analysis

The system allows access to a patients demographics and medical exam results to help support patient evaluation and treatment thereby improves healthcare professionals' work flow. The system also assists in accurate documentation of prescriptions and medications records to avoid transcription and calculation errors Automated data collection from monitors, ventilators and other equipment connected to the patient allows work flow optimization for healthcare professionals, so that they can concentrate on their primary function ie providing care to the patient. Real-time monitoring and storage of patient data also enables the scope for research and analysis. This will help in analysing the historical trends which will be very much useful in the future for providing better care. 


\section{CONCLUSION}

The existing clinical data entry and patient monitoring systems are not well organised and are not comprehensive. Our work reported here is an attempt to develop a more comprehensive system which in turn can relieve the clinical staff from lot of redundant activities. The Apps which are being developed focussing on ICU work flows will make life easier for them so that they will be able to concentrate on their primary objective that is to provide the required care. The conventional patient monitoring systems are not ubiquitous and thus will add to the delay in providing required care to the patient at the right time. Interfacing of bed side monitors to the central server will make the data available to the entire clinical team in a faster manner so that critical decisions are made faster.

The conventional alert generation systems are also not reliable since they look at individual parameters and generate an alert when the hard limits are crossed. The decision support system under development consisting of a classifier, context aware medical rule set, smart alarm generator can analyse the cross functional effects of different parameters and can generate alerts and possible advices based on a context aware rule set. These alerts will be generated well in advance so that care takers can provide medications earlier thereby saving the life of the critical patient.

\section{Acknowledgment}

The authors would like to thank the management and staff of Jubilee Medical Mission Hospital Thrissur, for allowing our team to implement the system at their Neuro ICU starting with workflow analysis, patient data collection, biomedical equipment interfacing leading to system design and implimentation.

We are indebted to M/s Mobilexion Technologies Pvt. Ltd., for allowing us to work on this project and providing all support for its implementation. We are indebted to our guide, Dr. George Varkey, for providing directions for the project and also in preparation of this paper.

\section{REFERENCES}

[1] Thongpithoonrat, Pongnarin; McKneely, Paul K.; Gumudavelli, Suman; Gurkan, D.; Chapman, Frank M. Networking and plug-and-play of bedside medical instruments. EMBS 2008; 1514 - 1517

[2] Mengling Feng; Zhuo Zhang; Feng Zhang; Yu Ge; Liang Yu Loy; Vellaisamy, K.; Wenyuan Guo; Pei Loon Chin; King,
N.K.K.; Beng Ti Ang; Cuntai Guan. iSyNCC: An intelligent system for patient monitoring and clinical decision support in Neuro-Critical-Care. IEMBS 2011; 6426 - 6429

[3] Nahm R, Poston I. Measurement of the effects of an integrated, point-of-care computer system on quality of nursing documentation and patient satisfaction. Comput Nurs 2000; 18:220229.

[4] Bosman RJ, Rood E, Oudemans-van Straaten HM, Van der Spoel JI, Wester JP, Zandstra DF. Intensive care information system reduces documentation time of the nurses after cardiothoracic surgery. Intensive Care Med.2003; 29:83-90.

[5] Joon Lee; Scott, D.J.; Villarroel, M.; Clifford, G.D.; Saeed, M.; Mark, R.G. Open-access MIMIC-II database for intensive care research. EMBC 2011: 83158318

[6] Apkon M, Singhaviranon P. Impact of an electronic information system on physician workflow and data collection in the intensive care unit. Intensive Care Med 2000; 27: 122130. 11.

[7] Insup Lee, Oleg Sokolsky, Sanjian Chen. Challenges and Research Directions in Medical Cyber-Physical Systems. Invited Paper in Special Issue on Cyber-Physical Systems, Proceedings of the IEEE, vol.100, no.1, pp.75-90, Jan. 2012.

[8] Shashi Gunawardane, Ravindra Koggalage, Ranga Rodrigo, Senaka Rajapakse. A Computer-Based ICU Patient Alert And Decision Support System. SLJCC , 2009, vol 1, 35-41.

[9] M. Borowski, M. Gorges, R. Fried, O. Such, C. Wrede, and M. Imhoff. Medical device alarms. Biomed Tech, 56:7383, 2011.

[10] S. Park, A. Roederer, R. Mani, S. Schmitt, P. D. LeRoux, L. H. Ungar, I. Lee, and S. E. Kasner. Limitations of thresholdbased brain oxygen monitoring for seizure detection. Neurocritical Care, April 2011.

[11] Fortier, P.; Jagannathan, S.; Michel, H.; Dluhy, N.; Oneill, E. Development of a hand-held real-time decision support aid for critical care nursing. HICSS 2003

[12] Yi Mao; Yixin Chen; Hackmann, G.; Minmin Chen; Chenyang Lu; Kollef, M.; Bailey, T.C. Medical Data Mining for Early Deterioration Warning in General Hospital Wards. ICDMW, 2011; 1042 - 1049

[13] Russell, L.; Steele, A.L.; Goubran, R. Low-cost, rapid prototyping of IMU and pressure monitoring system using an open source hardware design I2MTC, 2012; 269-2699 